\title{
Chronic Kidney Disease, CTCAE
}

National Cancer Institute

\section{Source}

National Cancer Institute. Chronic Kidney Disease, CTCAE. NCI Thesaurus. Code C143369.

A disorder characterized by gradual and usually permanent loss of kidney function resulting in renal failure. 